Research Paper

\title{
Efficient Inhibition of Human Glioma Development by RNA Interference-Mediated Silencing of PAK5
}

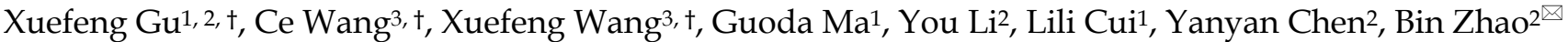 \\ and Keshen $\mathrm{Li}^{1}, 2 \bowtie$ \\ 1. Institute of Neurology, Guangdong Medical College, Zhanjiang, China. \\ 2. Guangdong Key Laboratory of Age-Related Cardiac and Cerebral Diseases, Affiliated Hospital of Guangdong Medical College, Zhan- \\ jiang, China. \\ 3. Department of Neurosurgery, The Fourth Affiliated Hospital of Harbin Medical University, Harbin, China. \\ † These authors contributed equally to this work.
}

\begin{abstract}
$\triangle$ Corresponding author: Keshen Li and Bin Zhao. Keshen Li, Institute of Neurology, Guangdong Medical College, Zhanjiang 524001, China. E-mail: ksl1971@163.com. Bin Zhao, Guangdong Key Laboratory of Age-Related Cardiac and Cerebral Diseases, Affiliated Hospital of Guangdong Medical College, Zhanjiang 524001, China. E-mail: zhaobine@vip.tom.com.
\end{abstract}

(C) 2015 Ivyspring International Publisher. Reproduction is permitted for personal, noncommercial use, provided that the article is in whole, unmodified, and properly cited. Please see http://ivyspring.com/terms for terms and conditions.

Received: 2014.03.23; Accepted: 2014.12.10; Published: 2015.01.12

\begin{abstract}
Glioma is the most common type of primary intracranial tumor and is highly lethal due to its pathogenetic location, high invasiveness, and poor prognosis. Even combined surgery and chemoradiotherapy do not effectively rescue glioma patients. Molecular target therapy is considered a safe and promising therapy for glioma. The identification of a novel, effective target protein in gliomas is of great interest. We found that PAK5 was highly expressed in the tumor tissues of glioma patients and human glioma cell lines. We then used a lentivirus-delivered short hairpin RNA to stably silence PAK5 expression in glioma cells and explore its influence. The results showed that the inhibition of PAK5 reduced cell viability and delayed the cell cycle at the G0/G1 phase in the glioma cells with PAK5 high expression. In addition, silencing PAK5 expression in U87 cells weakened their colony formation ability and in vivo tumorigenesis ability. Further studies demonstrated that PAK5 inhibition led to an increase in cleaved caspase 3 and a decrease in $\beta$-catenin. In conclusion, our results suggest that the inhibition of PAK5 by RNA interference might efficiently suppress tumor development of glioma cells with PAK5 high expression. This finding provides a novel, promising therapeutic target for glioma treatment.
\end{abstract}

Key words: glioma, PAK5, tumor development, RNA interference, cell cycle arrest

\section{Introduction}

Glioma is the most common type of primary brain tumor and accounts for approximately 46 percent of intracranial tumors. Its peak incidence occurs at 30-40 years of age. Glioma is the second leading cause of death in cancer patients less than 34 years of age [1]. With an annual incidence of 3-5/100,000 individuals, glioma is an infrequent malignancy, but its pathogenetic location, high invasiveness, and poor prognosis make glioma a highly lethal cancer. In spite of combined therapies of craniotomy, radiotherapy, and chemotherapy, the overall median survival period is approximately 15 months [2]. Effective glioma therapy remains a difficult challenge for medical professionals.

The investigation of the genes that influence glioma development will advance our understanding of the molecular pathogenesis of this deadly disease and, consequently, its treatment. The role of the p21-activated kinase (PAK) family in tumorigenesis has received increasing attention. PAKs are ser- 
ine/threonine kinases that function as downstream effectors in various oncogenic signaling pathways [3]. Members of the PAK family, which includes six isoforms, can be classified into two groups based on their sequences and functions. While the roles of group A PAKs (PAK1-3) in the signaling pathways of nervous system diseases have been thoroughly investigated, the functions of the group B PAKs (PAK4-6) remain unclear [4].

PAK5 expression is particularly high in the brain. In addition, PAK5 is generally expressed in nerve cells but not glial cells [5]. PAK5 can phosphorylate Raf1 at Ser338, which promotes translocation of Raf1 to the mitochondria [6]. In the mitochondria, Raf1 forms a complex with Bcl2 and further phosphorylates the pro-apoptotic protein BAD at the Ser112 site. Although Bcl-2 is an anti-apoptotic factor, the binding of $\mathrm{Bcl}-2$ to $\mathrm{BAD}$ induces the release of many pro-apoptotic proteins from the mitochondria and initiates apoptosis. The phosphorylation of BAD at Ser112 eliminates Bcl-2 binding [7]. PAK5 is overexpressed in some colorectal cancers and plays an important role in the migration of colorectal carcinoma cells [8]. PAK5 is also significant for neurite growth in neuroblastoma cells [9]. However, the role of PAK5 in gliomas remains unclear.

In this study, we determined that PAK5 expression is prominently higher in cancerous tissues of glioma patients than in pericancerous tissues. We used lentivirus-mediated short hairpin RNA (shRNA) to efficiently silence PAK5 expression in human glioma cells, which resulted in a decrease in cell viability and cell cycle arrest in the G0/G1 phase in glioma cells with PAK5 high expression. Colony formation and in vivo tumorigenesis were also reduced by PAK5 silencing in these glioma cells. PAK5 inhibition also caused an increase in cleaved caspase 3 and a decrease in $\beta$-catenin. Our findings demonstrate that inhibition of PAK5 by lentivirus-mediated RNA interference (RNAi) significantly suppresses tumor development in glioma cells with PAK5 high expression.

\section{Materials and Methods}

\section{Tissue Samples}

Samples of human glioma tissues and adjacent normal tissues were collected during the surgeries of 40 glioma patients. The pathologic features of the tumors were evaluated by WHO criterion. All patients provided written informed consent. No patients had previously received chemotherapy or radiotherapy. The samples were soaked in RNALater (Qiagen $\mathrm{GmbH}$, Hilden, Germany) for RNA extraction and preserved in $10 \%$ neutral-buffered formalin for histopathological and immunohistochemical analyses.
The study was approved by the Ethics Committee of the Affiliated Hospital of Guangdong Medical College, and the Helsinki Declaration of Human Rights was strictly observed.

\section{Immunohistochemistry}

Paraffin-embedded tissues were stained with hematoxylin and eosin to analyze morphological changes. The immunohistochemical antibodies were purchased from Abcam (Cambridge, MA, USA). The primary antibody was a PAK5 antibody (ab110069), and the secondary antibody was a goat polyclonal secondary antibody to rabbit IgG (HRP) (ab6721).

\section{Cell Culture}

The human glioma cell lines U87, SHG-44, CHG-5, and U251 were purchased from the American Type Culture Collection (ATCC, Manassas, VA, USA). U87, SHG-44 and U251 cells were cultured in Dulbecco's modified Eagle's medium (DMEM, GIBCO, Gaithersburg, MD) containing 10\% fetal bovine serum (FBS), $100 \mathrm{U} / \mathrm{ml}$ penicillin, and $100 \mu \mathrm{g} / \mathrm{ml}$ streptomycin at $37^{\circ} \mathrm{C}$ under humidified air containing $5 \%$ $\mathrm{CO}_{2}$. CHG-5 cells were cultured in Roswell Park Memorial Institute 1640 medium (RPMI-1640, GIBCO) under the same culture conditions as above.

\section{PAK5 silencing using lentivirus-delivered shRNA and PAK5 expressing using PAK5 vector}

Two shRNA candidates with PAK5 target sequences were used: PAK5-shRNA1 (5'-CGGG ATTACCACCATGACAAT-3') and PAK5-shRNA2 (5'-GCTCCTATGAAGACAATCGTT-3'). The sequence of the scrambled shRNA (Scr-shRNA) was 5'-TTCTCCGAACGTGTCACGT-3'. Scr-RNAi was used as a negative RNAi control. The oligonucleotides encoding the shRNA sequences were inserted into the GFP expression vector pGCL-GFP. The recombinant virus was packaged using Lentivector Expression Systems, and U87, SHG-44, and CHG-5 cells were infected. After 3 days, GFP-positive cells were counted under a fluorescence microscope (OLYMPUS, Japan). PAK5 expression after shRNA infection was determined by quantitative real-time PCR (qRT-PCR) or western blotting on the 4th day.

pCMV6-Myc-PAK5WT vector (Plasmid 16019) was purchased from addgene (Addgene Inc., Cambridge, MA, USA). PAK5 expression of infected U87 cells were determined by western blotting 3 days after cell infection.

\section{RNA extraction and $q R T-P C R$}

Total RNA was extracted from cells using TRIzol reagent (Invitrogen, Carlsbad, CA) and from tissues using RNALater according to the manufacturer's in- 
structions. cDNA was synthesized using a RevertAid First-Strand cDNA Synthesis Kit (Fermentas, Vilnius, Lithuania). Gene expression levels were detected by qRT-PCR using a standard SYBR Green RT-PCR Kit (Takara, Kyoto, Japan). The relative levels of the PAK5 gene mRNA transcripts were normalized to the control, GAPDH.

\section{Western blotting}

Cell lysates were subjected to SDS-PAGE. The blots were incubated with the desired primary antibodies, which included anti-PAK5 (Abcam, ab110069), anti-cleaved caspase 3, and anti- $\beta$-catenin (both from Cell Signaling Technology, Beverly, MA), followed by incubation with an anti-rabbit IgG peroxidase-conjugated secondary antibody (Abcam, ab6721) and chemiluminescent substrates. Hybridization with anti-GAPDH (Cell Signaling Technology) was used to confirm equal protein loading.

\section{Cell viability assay}

Cell viability was evaluated by a 3-[4,5-dimethylthiazol-2-yl]-2,5-diphenyl tetrazolium bromide (MTT) (Sigma Chemical, St. Louis, MO) assay. The cells of test groups were plated at a final concentration of 3,000 cells/well in 96-well culture plates for different culture times. Then, MTT (10 $\mu \mathrm{l}, 5$ $\mathrm{mg} / \mathrm{ml}$ ) was added to each well and incubated at $37^{\circ} \mathrm{C}$ for $1 \mathrm{~h}$. The reaction was terminated by replacing the MTT-containing medium with $100 \mu \mathrm{l}$ of DMSO, and the formazan salts were dissolved by gentle shaking for approximately $10 \mathrm{~min}$ at room temperature. The optical density (OD) of each well was measured at $490 \mathrm{~nm}$ using an ELISA reader (ELx808 Bio-Tek Instruments, USA).

\section{Cell cycle assay}

Cells were seeded into 6-well culture plates and harvested at 48 hours by centrifugation at $1200 \mathrm{rpm}$ for $5 \mathrm{~min}$. After washing twice with pre-cooled PBS (pH 7.4), the cells were fixed in $70 \%$ alcohol. The percentage of cells in each stage of the cell cycle was determined by staining with propidium iodide (PI, Sigma). The cell cycle distribution was analyzed using a flow cytometer (FACSCalibur, BD) in accordance with the manufacturer's guidelines.

\section{Colony formation assay}

Exponentially growing cells were suspended in complete growth medium and seeded in 6-well plates at 500 cells/well. The plates were maintained at $37^{\circ} \mathrm{C}$ in a humidified incubator with $5 \% \mathrm{CO}_{2}$ for one week. After fixation in paraformaldehyde, the colonies were stained with crystal violet for $10 \mathrm{~min}$, followed by imaging using a digital camera.

\section{Tumorigenesis assay}

Male nude mice (7-8 weeks old, weighing 18-20 g) were obtained from the Shanghai Experimental Center, Chinese Science Academy, Shanghai, and maintained at an animal facility under pathogen-free conditions. All animals received humane care according to the National Research Council's guidelines. U87 cells infected with Scr-shRNA or PAK5-shRNA lentivirus were resuspended in PBS at a final density of $1 \times 10^{7}$ cells $/ \mathrm{ml}$. A $100-\mu l$ aliquot of the cell suspension (equivalent to $1 \times 10^{6} \mathrm{U} 87$ cells) was injected into the axilla of the nude mice in the corresponding group. Some mice were sacrificed 3 weeks after injection, and tumor growth was examined. The other mice were used to detect the time of visible tumors (diameter $>3 \mathrm{~cm}$ ).

\section{Statistical methods}

The data were expressed as the mean \pm SEM. Statistical significance was determined using Student's t-test, one-way ANOVA and chi-square test using GraphPad Prism 5.0 software (GraphPad Software, San Diego, CA). A value of $\mathrm{P}<0.05$ was considered statistically significant.

\section{Results}

\section{Upregulation of PAK5 expression in gliomas}

PAK5 expression levels in human gliomas have not been previously determined, and thus, we first detected PAK5 mRNA expression in freshly sampled gliomas and in matched pericancerous tissues from 40 glioma patients. The data suggested that PAK5 mRNA expression is markedly higher in cancerous tissue than in pericancerous tissue in most (35 to 40) glioma patients (Fig. 1A). To confirm the high expression of PAK5 in gliomas, we performed immunohistochemical analysis to evaluate PAK5 expression in human gliomas. The glioma tissue exhibited strong positive staining by the anti-PAK5 antibody, whereas PAK5 expression levels were low in most pericancerous tissues of glioma patients (Fig. 1B). However, PAK5 expression had no obvious correlations with grades of glioma tumors (Table 1). On the other hand, immunoblot analysis demonstrated that PAK5 expression levels are high in human glioma cell lines, particularly in U87 and SHG44 cells (Fig. 1C). Taken together, these data suggest that PAK5 expression is upregulated in human gliomas. 
Table 1. Associations between PAK5 expression and clinicopathological characteristics of 40 glioma patients

\begin{tabular}{llllllll}
\hline \multicolumn{2}{l}{$\begin{array}{l}\text { Clinicopathological } \\
\text { parameters }\end{array}$} & $\mathbf{n}$ & down & $\mathbf{0 - 1}$ & $\mathbf{1 - 2}$ & $\mathbf{> 2}$ & P value \\
\hline age & $<60$ & 32 & 5 & 2 & 5 & 20 & $>0.05$ \\
& $>60$ & 8 & 1 & 0 & 2 & 5 & \\
\multirow{2}{*}{ gender } & male & 26 & 3 & 1 & 4 & 18 & $>0.05$ \\
& female & 14 & 3 & 1 & 3 & 7 & \\
grade & I-II & 16 & 3 & 1 & 3 & 9 & $>0.05$ \\
& III-IV & 24 & 3 & 1 & 4 & 16 & \\
\hline
\end{tabular}

$P$ value was detected by chi-square test

\section{Lentivirus-delivered targeted RNAi stably in- hibits PAK5 expression in human glioma cells}

While normal glial cells do not express PAK5 [5], our results indicated that glioma cells express high levels of PAK5. This difference between normal glial cells and glioma cells suggests that PAK5 expression might affect glioma development. To investigate the role of PAK5 in glioma tumorigenesis, we used lenti-

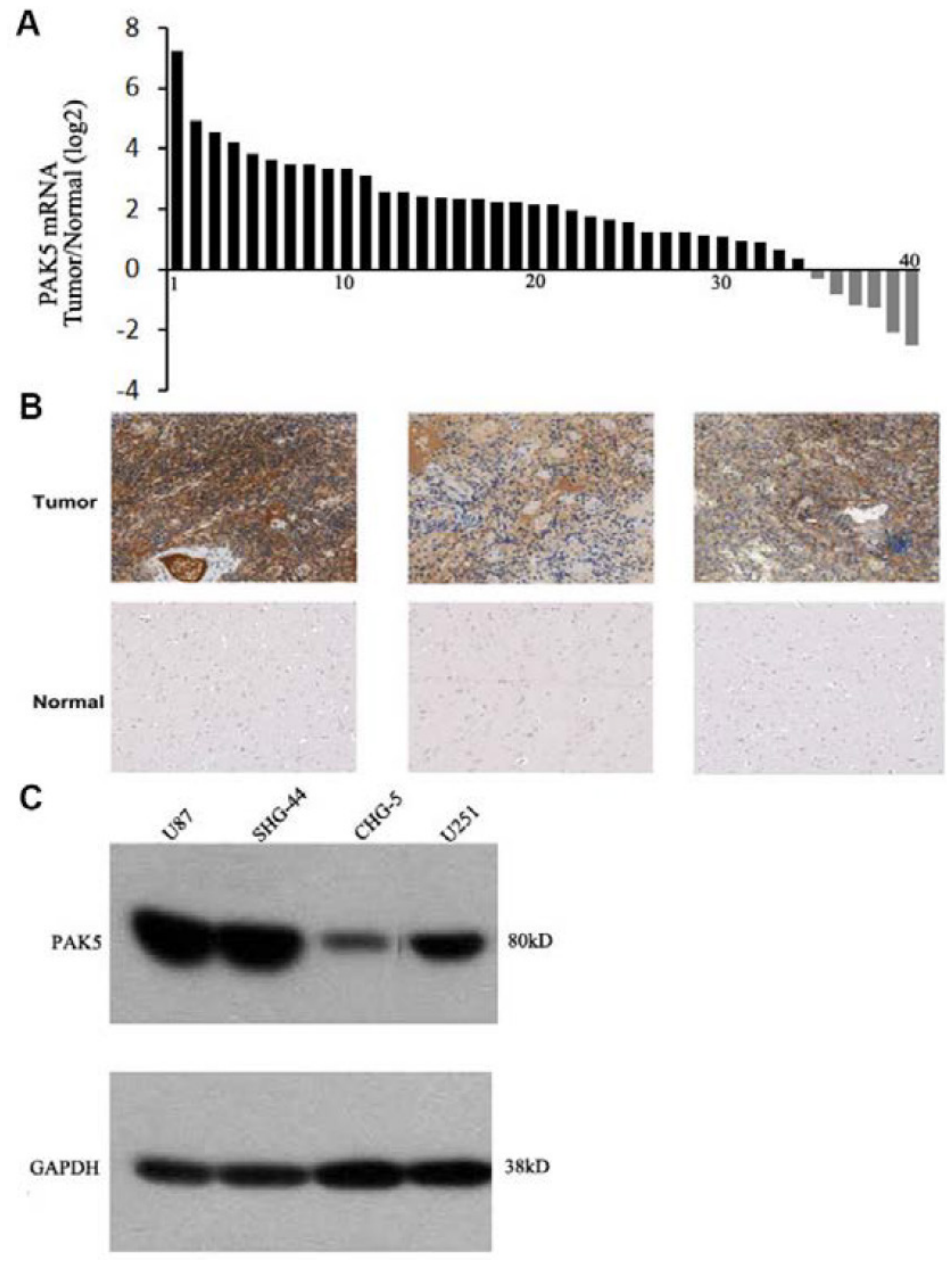

Figure 1. PAK5 is highly expressed in glioma cells. (A) The ratio of PAK 5 mRNA expression in freshly sampled gliomas and their adjacent normal tissues from 40 glioma patients. (B) Representative immunohistochemical staining of PAK5 expression in human glioma tissues (upper) and pericancerous tissues (lower). (C) Western blot assay of PAK5 expression in glioma cell lines. virus-mediated RNAi to silence PAK5 expression in a human glioma cell line. Lentiviral vectors expressing PAK5-specific shRNA and Scr-shRNA were used to infect U87 cells. After three days, high-efficiency infection was observed (Fig. 2A). To determine the effectiveness of the inhibition of PAK5 expression by the PAK5-shRNA-expressing lentiviral vectors, we detected PAK5 mRNA and protein levels in infected cells by real time-PCR and western blot. Both PAK5-shRNAs significantly reduced PAK5 mRNA expression (Fig. 2B). In addition, PAK5 protein levels were also prominently decreased in the two PAK5-shRNA-infected groups compared with the Scr-shRNA infected group (Fig. 2C). In addition, SHG-44 and CHG-5 cells also showed the similar results (Fig. 2D and E). Therefore, both types of PAK5-shRNA efficiently downregulated PAK5 expression in human glioma cells.

\section{Inhibition of PAK5 significantly augments tu- mor growth in glioma cells with PAK5 high expression}

We next explored the effects of PAK5 inhibition in glioma cells. First, we used a MTT assay to assess cell growth dynamics in Scr-shRNA- and PAK5-shRNA-infected cells. After 5 days, in U87 and SHG-44 cells, PAK5-shRNA-infected cells displayed remarkable cell growth inhibition compared with Scr-shRNA-infected cells. At day 5, the cell viability of the Scr-shRNA-infected group was 4 times higher than that of the PAK5-shRNA-infected groups (Fig. 3A and $B)$. However, there was no significant difference between Scr-shRNA- and PAK5-shRNA-infected CHG-5 cells, cells with low PAK5 expression (Fig. 3C). To further determine the effect of PAK5 in glioma cells with high PAK5 expression, we enhanced PAK5 expression by transfecting PAK5 vector in PAK5-shRNA1-infected U87 cells (Fig. 3D). The results showed that enhancing PAK5 expression rescued the decrease of cell viability resulting from RNAi-mediated PAK5 knockdown (Fig. 3E).

Flow cytometric analysis of cell cycle of U87 cells by PI staining revealed that PAK5 inhibition resulted in an increase in cells detained at the G0/G1 phase and a decrease in cells at the G2/M phases (Fig. 4B). These data demonstrate that PAK5 inhibition forces U87 cells into the quiescent phase. Moreover, the colony-forming ability of the PAK5-shRNA infected groups also obviously decreased, in contrast to the Scr-shRNA infected group 
(Fig. 4A). These data demonstrate that the inhibition of PAK5 expression decreases U87 cell growth in vitro. A nude mouse tumorigenesis test revealed that the tumorigenesis ability of U87 cells was also weakened by silencing of PAK5 expression (Fig. 4C and D). Taken together, these results suggest that the inhibition of PAK5 expression suppresses tumor growth in glioma cells with PAK5 high expression.

\section{PAK5 silencing causes caspase 3 activation and the downregulation of $\beta$-catenin}

Next, we examined the mechanism by which PAK5 inhibition suppresses glioma growth. Role of PAK5 in tumor development has been poorly described, and some studies have reported that PAK5 has an anti-apoptotic function that is dependent on AKT activation [7, 10]. Therefore, we primarily focused on AKT-associated signaling pathways. Western blot analysis revealed that cleaved caspase 3 levels were higher in PAK5-shRNA-infected cells than in Scr-shRNA-infected cells (Fig 5), indicating that PAK5 silencing promotes cell apoptosis in glioma cells. However, our above results demonstrated that PAK inhibition results in cell cycle arrest in the G0/G1 phase (Fig. 4A). Therefore, we also analyzed the levels of proteins in the AKT-associated cell cycle pathway, which revealed that $\beta$-catenin protein levels were significantly reduced by PAK5 inhibition in glioma cells (Fig. 5). $\beta$-catenin might promote the expression of cyclin D1, thereby contributing to cell cycle progression [11]. The decrease in the $\beta$-catenin level may lead to cell cycle arrest in glioma cells. Moreover, enhancing PAK5 expression reverse this trend (Fig 5). These results demonstrated that silence of PAK5 silencing activated caspase 3 and downregulated $\beta$-catenin in the glioma cells.
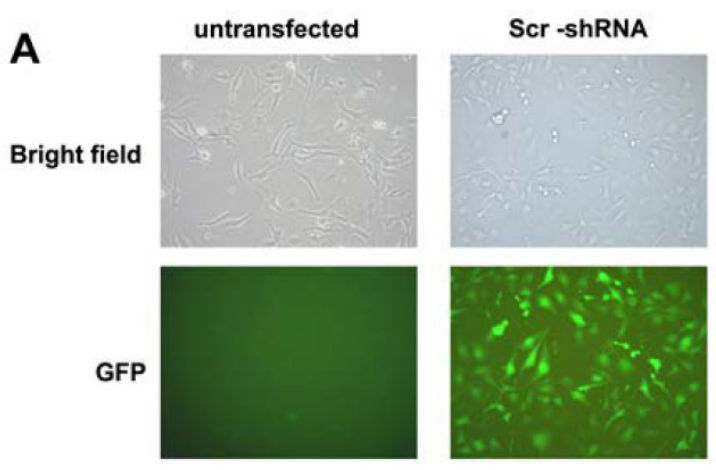

B

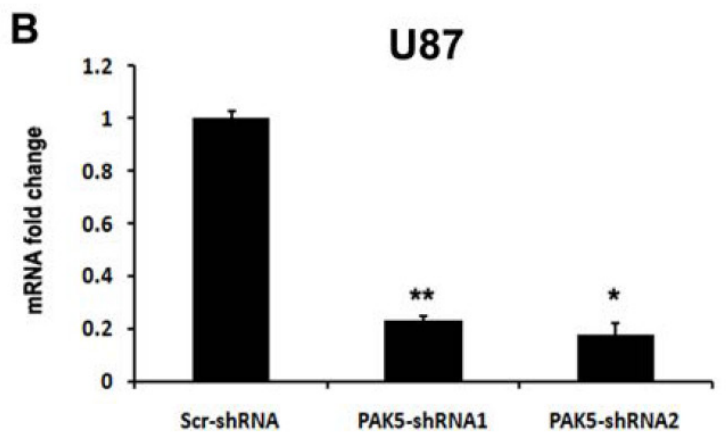

D

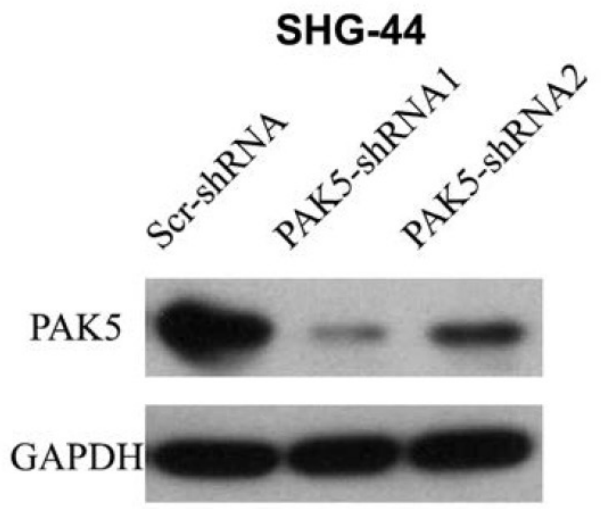

PAK5-shRNA1
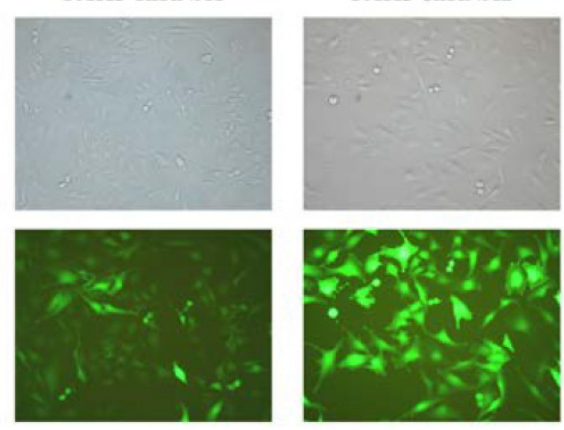

C

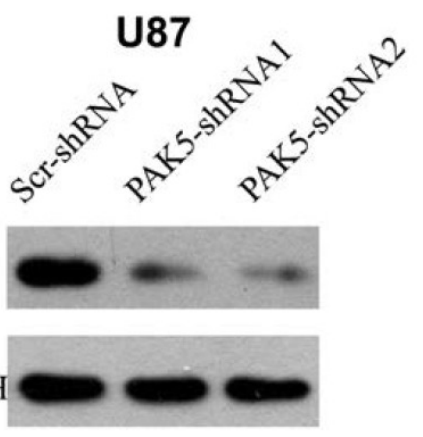

E
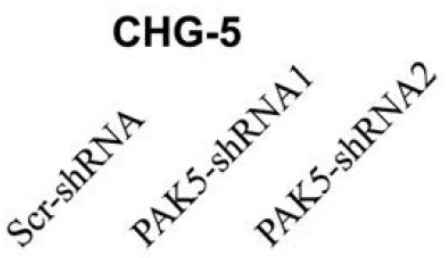

PAK5

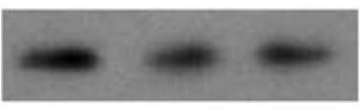

GAPDH
Figure 2. Lentivirus-mediated RNAi significantly inhibits PAK5 expression in human glioma cells. (A) The lentiviral transduction efficiency in U87 cells was estimated 3 days after infection at an $\mathrm{MOI}$ of 5 . Light micrograph (upper); fluorescent micrograph (lower) (× 400). (B) Total RNA of U87 cells was extracted at 4 days after infection, and relative PAK5 mRNA expression was determined by qRT-PCR. GAPDH was used as an internal standard. The data represent the mean \pm SEM of three independent experiments. *, $\mathrm{P}<0.05$, **, $\mathrm{P}<0.01$. (C-E) Western blot analysis of PAK5 protein expression levels of Scr-shRNA-infected and PAK5-shRNA-infected cells in U87 (C), SHG-44 (D) and CHG-5 (E) cells. 
A

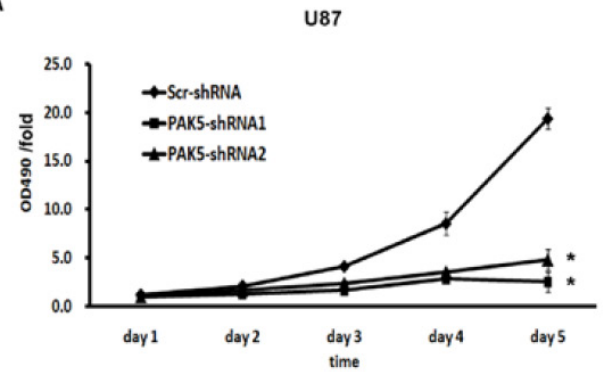

B

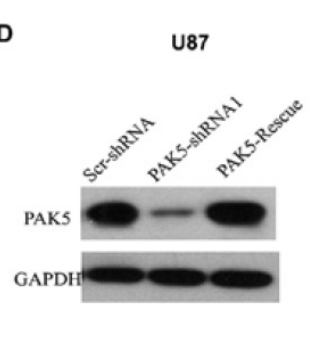

SHG-44

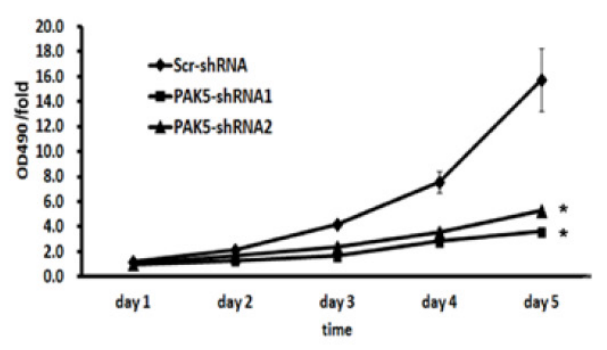

E
C

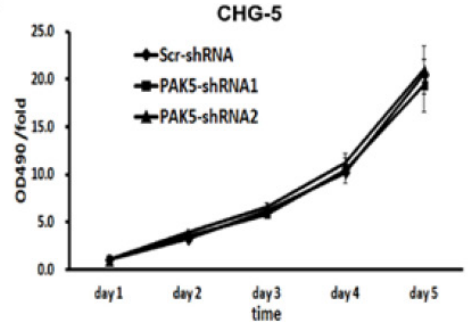

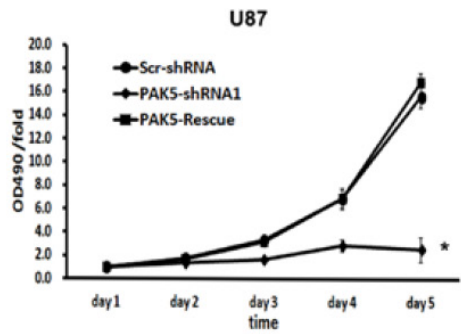

Figure 3. Inhibition of PAK5 remarkably suppresses cell viability in glioma cells with high PAK5 expression. (A-C) Cell viability curves of U87 (A), SHG-44 (B) and CHG-5 (C) cells during the 5 days were evaluated by the MTT assay. The data represent the mean \pm SEM of three independent experiments. *, P $<0.05$ vs. cells infected with Scr-shRNA lentivirus. (D) PAK5 protein expression in U87 cells infected with a vector with expressing PAK5 after PAK5-shRNA1-mediated PAK5 knockdown. (E) Cell viability curves of U87 cells of three groups during the 5 days were evaluated by the MTT assay. The data represent the mean \pm SEM of three independent experiments. *, P $<0.05$ vs. cells infected with Scr-shRNA lentivirus.

\section{Discussion}

Gliomas are the most malignant brain tumors, and glioma patients do not survive more than a few months after diagnosis, even when treated with a combination of surgery, radiotherapy, and chemotherapy. As the in-depth understanding of the molecular mechanisms of glioma development has improved, molecular targeted therapy has been proposed for the treatment of gliomas. EGFR [12], PTEN [13], and PDGFRa [14] have important roles in glioma development and have been identified as protein targets for glioma treatment. However, therapies that target these proteins and their associated pathways do not completely cure glioma [15], and thus, novel therapeutic targets are urgently needed.

In the present study, high PAK5 expression was observed in glioma tissue from glioma compared to adjacent normal tissues. The in vitro study also revealed that PAK5 was expressed at high levels in most human glioma cell lines. We used lentivirus-delivered specific shRNAs to efficiently silence PAK5 expression in human glioma cells. Inhibition of PAK5 significantly resulted in the decrease of cell viability in the glioma cells with PAK5 high expression. Moreover, PAK5 inhibition led to cell cycle arrest in the G0/G1 phase in U87 cells. In addition, U87 cells infected with PAK5-shRNAs had prominently reduced colony formation and in vivo tumorigenesis ability. Further examination revealed that silencing PAK5 resulted in an increase in cleaved caspase 3 levels and a decrease in $\beta$-catenin levels in U87 cells. These re- sults suggest that inhibition of PAK5 by lentivirus-mediated RNAi efficiently suppresses tumor development in the glioma cells with PAK5 high expression.

In the brain, PAK5 is constitutively expressed in nerve cells but not in glial cells [5]. However, our data revealed high PAK5 expression levels in glioma tissue but no detectable expression in the matched pericancerous tissues. Thus, PAK5 may play an important role in glioma development. PAK5 is one of the most poorly understood PAK family isoforms. Previous studies have reported a role for PAK5 in inhibiting cell apoptosis $[7,16]$, and PAK5 overexpression promotes neurite growth in a neuroblastoma cell line [9]. In the present study, we determined that silencing PAK5 increased cell apoptosis in glioma cells. The AKT pathway plays an important role in the PAK5-mediated anti-apoptotic pathway [6]. In addition, Mdm2, which is a downstream gene of AKT, is highly expressed in malignant human glioma cells [17]. However, we determined that PAK5 inhibition had no impact on the level of the anti-apoptotic protein Bcl-2, which is upregulated by activation of the AKT/Mdm2 pathway (data not shown). By contrast, our results demonstrated that PAK5 inhibition restrained the cell cycle at the G0/G1 phases. Han ZX, et al. also demonstrated that suppression of PAK5 in human glioma cell lines led to G1 phase arrest and the increase of cell apoptosis [18]. The mechanism underlying this effect may involve the decrease in $\beta$-catenin induced by PAK5 inhibition (Fig. 4). $\beta$-catenin is upregulated by the AKT/GSK3 $\beta$ path- 
way, which thereby triggers the cell cycle by enhancing cyclin D1 expression [11]. Additionally, the study of Han ZX et al. also showed that PAK 5 played an important role in cell migration and invasion potentially through PAK5-Egr1-MMP2 signaling pathway [18]. Notably, inhibition of PAK5 had no obviously effect on the glioma cell with PAK5 low expression
(Fig. 3C). The reason may be that these cells have been screening during the process of tumor development and these screened cells have already activated some compensatory PAK5-independent pathway to support cell survival and proliferation. However, the exact mechanisms need to further explore.
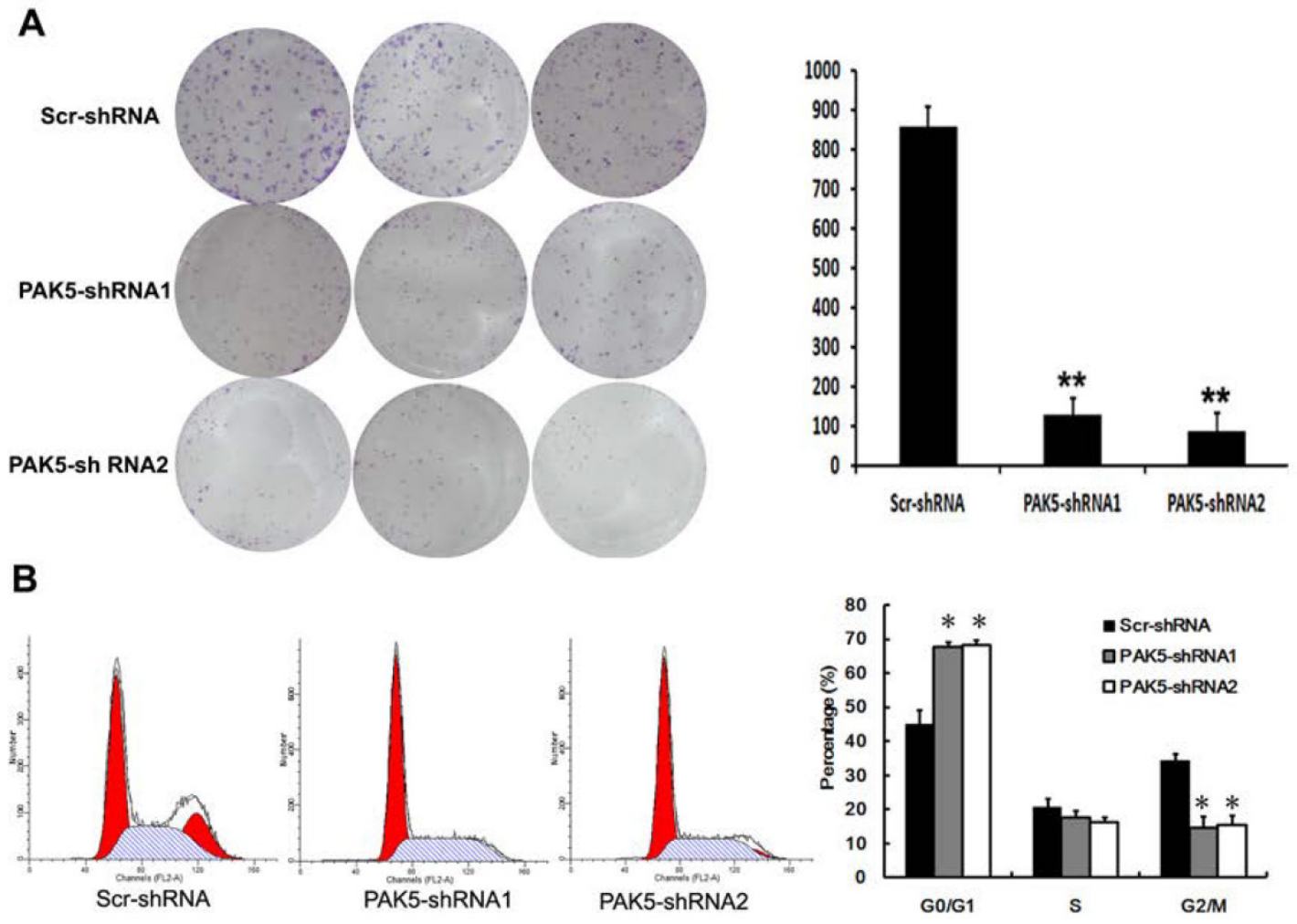

C

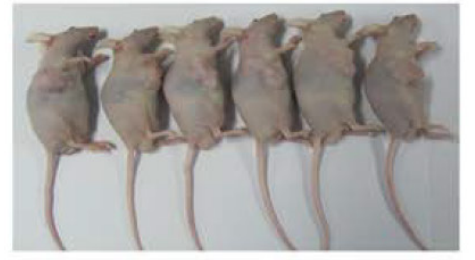

Scr-shRNA

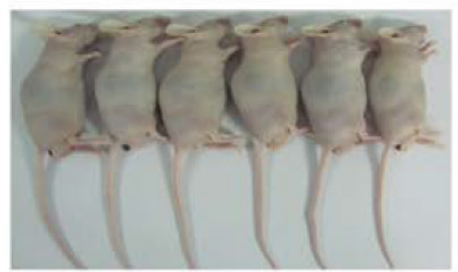

PAK5-shRNA2

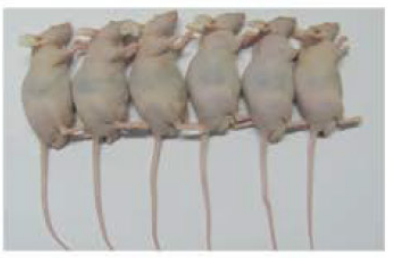

PAK5-shRNA1
D

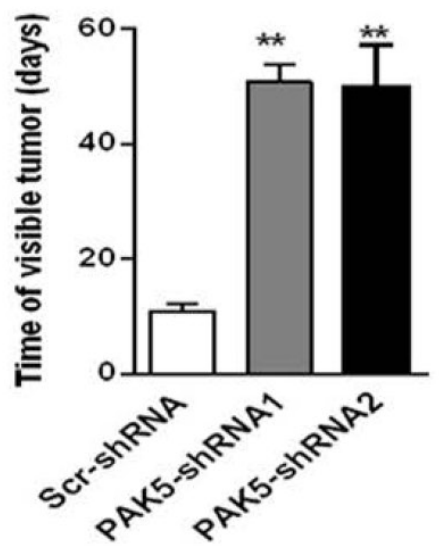

Figure 4. Inhibition of PAK5 prominently suppresses tumor development in U87 cells. (A) Colony formation assay of cells from the three groups. Cells were seeded at 500 cells/well and allowed to form colonies for 7 days. The colonies were stained with crystal violet and counted. Data represent the mean \pm SEM of three independent experiments (right). ${ }^{* *}, \mathrm{P}<0.01$. (B) Cell cycle analysis of cells from the three groups as determined by $\mathrm{PI}$ staining and FACS analysis (left). The percentages of cell cycle phases represent the mean $\pm \mathrm{SEM}$ of three independent experiments (right). *, $\mathrm{P}<0.05$. (C, D) Scr-shRNA-infected and PAK5-shRNA-infected groups of nude mice were injected with the corresponding infected U87 cells (106 cells per mice) into the axilla. After 3 weeks, some mice were sacrificed, and their tumors were examined $(n=6)(C)$. The other mice were used to detected the time of visible tumors (diameter $>3 \mathrm{~cm})(\mathrm{n}=6)(\mathrm{D})$. $* *, \mathrm{P}<0.01$. 


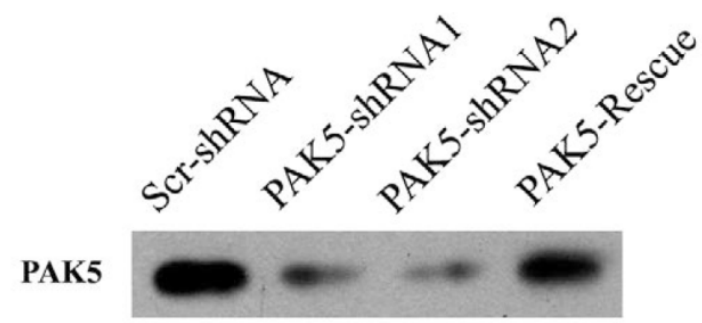

Cleaved Caspase-3

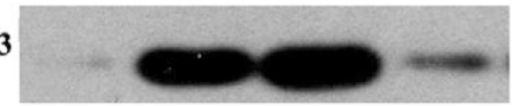

$\beta$-catenin

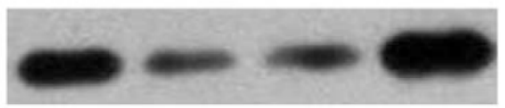

GAPDH

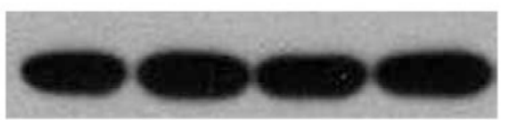

Figure 5. PAK5 silencing increases cleaved caspase 3 levels and decreases $\beta$-catenin levels in U87 cells. Cell lysates of Scr-shRNA-infected, PAK5-shRNA-infected and PAK5-shRNA1-PAK5 vector-co-infected U87 cells were immunoblotted with the indicated antibodies.

Taken together, our results suggest that PAK5 is overexpressed in human glioma tissues. Moreover, the inhibition of PAK5 by lentivirus-mediated RNAi suppressed glioma development by weakening the anti-apoptotic ability of cells and promoting cell cycle arrest. This study provides a novel therapeutic target for gliomas, although the molecular mechanisms of PAK5 in gliomas require further investigation.

\section{Abbreviations}

PAK, p21-activated kinase; shRNA, short hairpin RNA; DMEM, Dulbecco's modified Eagle's medium; FBS, fetal bovine serum; RPMI-1640, Roswell Park Memorial Institute 1640 medium; Scr-shRNA, scrambled shRNA; qRT-PCR, quantitative real-time PCR; MTT, 3-[4,5-dimethylthiazol-2-yl]-2,5-diphenyl tetrazolium bromide; OD, optical density

\section{Acknowledgments}

This work was supported by the funding: the National Nature Science Foundation of China (No. 31171219, 81271213, 81070878, 81271214 and 81261120404), the Natural Science Foundation of Guangdong Province (No. S2012010008222), the Science and Technology Innovation Fund of Guangdong Medical College (No. STIF 201101) and Natural Science Foundation of Heilongiiang Province, China (D201257).

The authors thank Dawn S. for critical reading and editing of the manuscript.

\section{Competing Interests}

The authors have declared that no competing interest exists.

\section{References}

1. Wen PY, Kesari S. Malignant gliomas in adults. $N$ Engl J Med. 2008;359:492-507.

2. Preusser M, de Ribaupierre S, Wohrer A, Erridge SC, et al. Current concepts and management of glioblastoma. Ann Neurol. 2011; 70: 9-21.

3. Daniels RH, Bokoch GM. p21-activated protein kinase: a crucial component of morphological signaling? Trends Biochem Sci. 1999;24:350-5.

4. Arias-Romero LE, Chernoff J. A tale of two Paks. Biol Cell. 2008;100:97-108.

5. Li X, Minden A. Targeted disruption of the gene for the PAK5 kinase in mice. Mol Cell Biol. 2003;23:7134-42.

6. Wu X, Carr HS, Dan I, Ruvolo PP, Frost JA. p21 activated kinase 5 activates Raf-1 and targets it to mitochondria. J Cell Biochem. 2008;105:167-75.

7. Cotteret S, Jaffer ZM, Beeser A, Chernoff J. p21-Activated kinase 5 (Pak5) localizes to mitochondria and inhibits apoptosis by phosphorylating BAD. Mol Cell Biol. 2003;23:5526-39.

8. Gong W, An Z, Wang Y, Pan X, Fang W, Jiang B, et al. P21-activated kinase 5 is overexpressed during colorectal cancer progression and regulates colorectal carcinoma cell adhesion and migration. Int J Cancer. 2009;125:548-55.

9. Dan C, Nath N, Liberto M, Minden A. PAK5, a new brain-specific kinase, promotes neurite outgrowth in N1E-115 cells. Mol Cell Biol. 2002;22:567-77.

10. Wang X, Gong W, Qing H, Geng Y, Zhang Y, Peng L, et al. p21-activated kinase 5 inhibits camptothecin-induced apoptosis in colorectal carcinoma cells. Tumour Biol. 2010;31:575-82.

11. Clevers H, Nusse R. Wnt/beta-catenin signaling and disease. Cell. 2012; 149: 1192-205.

12. Libermann TA, Nusbaum HR, Razon N, Kris R, Lax I, Soreq H, et al. Amplification, enhanced expression and possible rearrangement of EGF receptor gene in primary human brain tumours of glial origin. Nature. 1985;313:144-7.

13. Li J, Yen C, Liaw D, Podsypanina K, Bose S, Wang SI, et al. PTEN, a putative protein tyrosine phosphatase gene mutated in human brain, breast, and prostate cancer. Science. 1997; 275: 1943-7.

14. Hermanson M, Funa $\mathrm{K}$, Hartman $\mathrm{M}$, Claesson-Welsh L, Heldin $\mathrm{CH}$, Westermark B, et al. Platelet-derived growth factor and its receptors in human glioma tissue: expression of messenger RNA and protein suggests the presence of autocrine and paracrine loops. Cancer Res. 1992;52:3213-9.

15. Sathornsumetee S, Reardon DA, Desjardins A, Quinn JA, Vredenburgh JJ, Rich JN. Molecularly targeted therapy for malignant glioma. Cancer. 2007; 110: 13-24.

16. Cotteret S, Chernoff J. Nucleocytoplasmic shuttling of Pak5 regulates its antiapoptotic properties. Mol Cell Biol. 2006;26:3215-30.

17. Ranuncolo SM, Varela M, Morandi A, Lastiri J, Christiansen S, Bal de Kier Joffe E, et al. Prognostic value of Mdm2, p53 and p16 in patients with astrocytomas. J Neurooncol. 2004;68:113-21.

18. Han $Z X$, Wang $X X$, Zhang $S N$, Wu JX, Qian HY, Wen $Y Y$, et al. Downregulation of PAK5 inhibits glioma cell migration and invasion potentially through the PAK5-Egr1-MMP2 signaling pathway. Brain Tumor Pathol. 2014;31:234-41. 